Automation and Robotics in Construction XII

E.Budny, A.McCrea, K.Szymanski (Editors)

151

(C) 1995 IMBiGS. All Rights reserved

\title{
Automated Routing and Obstruction Detective System for Trench Construction : A GIS Approach
}

Min-Yuan Cheng ${ }^{1}$ and Guey-Lin Chang ${ }^{2}$

${ }^{1}$ Department of Construction Engineering \& Technology, National Taiwan Institute of Technology, Associate Professor

${ }^{2}$ Department of Construction Engineering \& Technology, National Taiwan Institute of Technology, Research Assistant

\begin{abstract}
Unlike most civil engineering projects, trenching schemes are not confined to a recognized site but, by their nature, connect distant locations and involve working in a variety of rural and urban environments. In trench construction, one of the tasks for the engineers is to select a suitable route to minimize construction cost and obstructions. This paper develops a GIS based system to automate the process of routing and design of the underground electric distribution supply system. In the system, the ground surface and underground utilities are represented in several data layers (coverages). Each data layer is integrated with a relational database. Using network analysis, the system determines the optimum paths for routing the underground utilities. Through database analysis and spatial operations, the construction conflict points between the basic utilite layout and the selected route identified. The primary features of the system are as follows: (1) identify the routing alternatives along the existing streets, (2) determine the optimal position and dimensions of the underground utilities to minimize the influence on the existing public utilities and structures, (3) integrate construction schedule and design information to identify the rellocation schedule of the existing public utilities, an' (4) provide the distribution information of the existing underground utilities to reduce potential damages or fractures caused by construction excavation. This system improves the trench routing and construction efficiency by providing a logical and systematic manner for integrating construction considerations in design phase. Furthermore, compared with manual methods, the system significantly improves the computational effort and increases the data accuracy and consistency.
\end{abstract}

\section{INTRODUCTION}

For most tunnel projects of the electrical supply system, the construction sites widely spread over the rural and urban areas, and are often in close contact with existing public utilities and structures. The underground net of most public utilities consist of live systems and principal services, water and gas can be dangerous to trench operatives when damaged or fractured. Especially, in urban area, obstacles such as existing 
underground public utilities, railways, canals, motorways can influence the route significantly as there may only be a limited number of feasible crossing points. Thus, selecting a suitable route to avoid existing obstacles can not only reduce the risk of damaging the existing utilities, but also the cost and duration required for construction.

The primary drawback of current planning methods is that the information required for trench route planning and design is incomplete and stored in different administrative authorities of the city government. Currently, the historical data of the existing public utilities such as pipe distributions, pipe types, and pipe dimensions is not propely recorded and managed. Various types of utilities are managed by different city government authorities. The coordination between the different utility administrations is very poor. Thus, for a trench project, the information required for route path planning is limited and the designer has to perform his work based on incomplete information. In the construction phase, due to the lack of accurate existing underground utility information, the constructor is apt to damage and fracture the existing utilities and cause the construction delay. The conventional approaches of the electricity route planning relies on heuristic strategies and rules of thumb based on the individuals' knowledge and experience gained from previous solution to the similar problem. In addition, the complexity of the problem involves the need to process spatial data such as road and circuit maps, records of the public utilities, and construction plans. In the classic manual approach, the planner has difficulth in identifying the spatial relationship between different data layers and usually results in an overdesigned, costly, and nonstandardized solution.

Summarizing the drawbacks of the current approach for electricity route planning and design, Geographic Information Systems (GIS), a technology for spatial data analysis, seems to have significant potential in solving these problems. Using GIS, planners can integrate and organize ill-structured construction information such as circuit maps and records, road maps and associated attributes, construction maps, and other spatial information more efficiently. Owing to its spatial operation capability, GIS helps planners to identify the spatial relationships between different data layers. GIS can not only store the spatial features and attributes, but it can be used as an analysis tool. GISArc/Info provides an internal macro language for users to automate their system design and the ability to interface with other application softwares. Hence, heuristic methods and algorithms can be developed to solve the route planning and design of the previously ill-structured problem.

\section{RESEARCH OBJECTIVES}

The primary purpose of this paper is to investigate the potenial for automating the process of routing and design of the underground public utility system. The objectives required to achieve the primary purpose are the following: (1) identify the routing alternatives along the existing streets, (2) determine the optimal position and dimensions of the underground utilities to minimize the influence on the existing public utilities and structures, (3) identify the conflict points between the designed route and the existing public utilities, (4) integrate construction schedule and design information to identify the reallocation schedule of the existing public utilities, and (5) provide the distribution information of the existing underground utilities to reduce potential damages or fractures caused by construction excavation. 


\section{SCOPE DEFINITION}

The system, RoutePlan, is developed to assist engineers to select a suitable route to minimize construction cost and obstructions. In this study, the system is mainly focused on the route planning and design of the electric utility. The open trench method is the construction method used for excavation. However, using the same methodology and algorithms with appropriate modifications, RoutePlan can be applied to different public utility design and construction methods.

\section{SYSTEM DEVELOPMENT}

\subsection{Knowledge Acquisition}

Knowledge Resources: This study reviewed the field practice and identified contemporary state of art route planning. To obtain the expert's knowledge and experience in electric utility route design, four resources were compiled and reviewed: (1) interview with experts, (2) previous research works, (3) design hand books, and (4) construction practice.

Rules for Route Planning: Due to the designer's experience, regulation restrictions and the complexity of the problem, the determination of the optimal route may vary from project to project. Most of the route designs are based on individuals' experience and common sense gained from the previous projects. However, there still exists some ruleof-thumb practices in construction management for carrying out route planning. Therefore, in this study, the knowledge and experience for route planning was compiled into two categories: (1) primary rules, and (2) specific concerns. The primary rules are the general rules that can be applied to most utility routing and design, while the specific concerns is the unique aspect of trench excavation in urban areas.

\subsection{System Functions}

The functional modules of the system are developed according to the capabilities and information needed for the user to conduct the route planning and design. The primary functions of the system are described as follows:

* Graphical display: The graphical display is a graphic representation of the site features and the associated tabular attributes. A communication and various functions are the decision support system which assists the user to identify the distributions of the existing public utilities, conflict points, and the optimal route. Depending on the user needs, the site coverages and the analysis results can be displayed and overlaid on the screen in either a detailed or abstracted form. The querying of the tabular attributes is the function used to display the labels associated with the site coverage and the integrated information of a particular entity in the coverage.

* Database query: The related databases of the site coverages can be identified using the database query. The main difference between database query and graphical display is that the database query can manipulate and list the integrated information of a coverage without displaying the site features. For example, without opening the road coverage, user can query and list the attributes of the road coverage such as road width, number of lanes, lane width, road condition, traffic condition etc. 
* Spatial analysis: The spatial analysis module is the function to identify the spatial relationships between the site features and the potential routes. The six Arc/Info's OVERLAY functions, namely Reselect, Buffer, Erasecov, Union, Intersect, and Identity, provided in this module allows the user to conduct the route planning manually.

* Route planning: The Route Planning module is a function which allows the user to identify a suitable route for design. Four primary functions, viz., potential route analysis, optimal route selection, locate conflict points, and reallocation schedule, are provided to achieve the task of route planning and design.

\subsection{System Design and Development Algorithm}

The system consists of knowledge and databases specific to the utility route planning and algorithms for integrating and automating the route design. The system, as shown in Figure 1, is an integration of GIS and database management systems. The algorithm of the system is primary divided into four parts, viz., potential route analysis, determination of the optimal route, locate the conflict points, and identification of the reallocation schedule of the existing utilities. The system design flow is discussed as follows:

Potential routes analysis: This section discusses the process of analysis of the potential routes. An Arc/Info dictionary including a list of coverages, the names of the attributes, and a description of the attribute values is created for reference during the development of the Arc/Info project database. After creating the site geometry and modeling the coverages, Analytical Hierarchy Process (AHP) method is used to identify the factors that influence the selection of the routes. Also, the weight of the factors is determined. Using the concept of Searching by Elimination, the system develops an algorithm for analyzing the potential routes between two nodes in the road network (see Figure 2). Considering the constraints and selection criteria, the routes without meeting the requirements are eliminated from the network.

Determination of the optimal route: The primary function of optimal route selection is to identify the route of least impedance between two nodes in the road network. The route selection process of the system is developed using the Arc/Info's Network functions. Initially, the dominant factors and the weight of the factors are identified and added to the road coverage related database. The weight of each road is the sum of the product of the factors and the weight of the factors. Then, an objective function called Impedance Index (II) is developed to determine the optimal route. The II is calculated by summing the product of the distance and the weight of the road. The route with least impedance between two nodes is the optimal route.

Locate the conflict points: This section is to identify the conflict points between the selected route and the existing public utilities. The selected route is first buffered based on the width of excavation. Using the Arc/Info's Overlay functions, the conflict points between the selected route and the existing facilities such as underground public utilities, railways, canals, motorways are identified.

Identification of the reallocation schedule of the existing utilities: Based on the construction progress and the distance from the start node of the selected route to the identified conflict point, the reallocation schedule of the existing utilities is identified. In the system, Excel macro is used to calculate the distance between two nodes and the schedule for reallocation. 


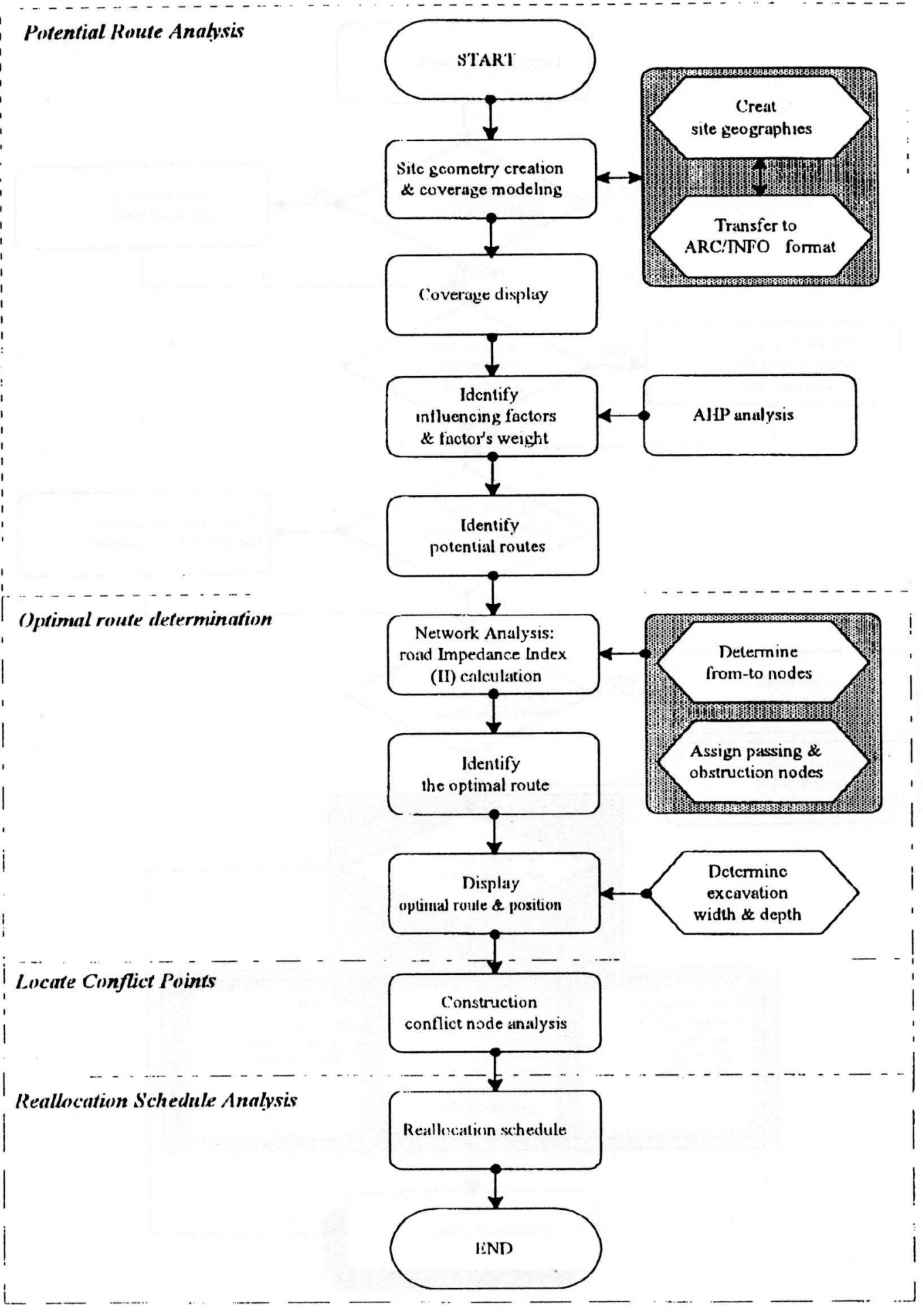

Figure 1 System Design and Development Algorithm 


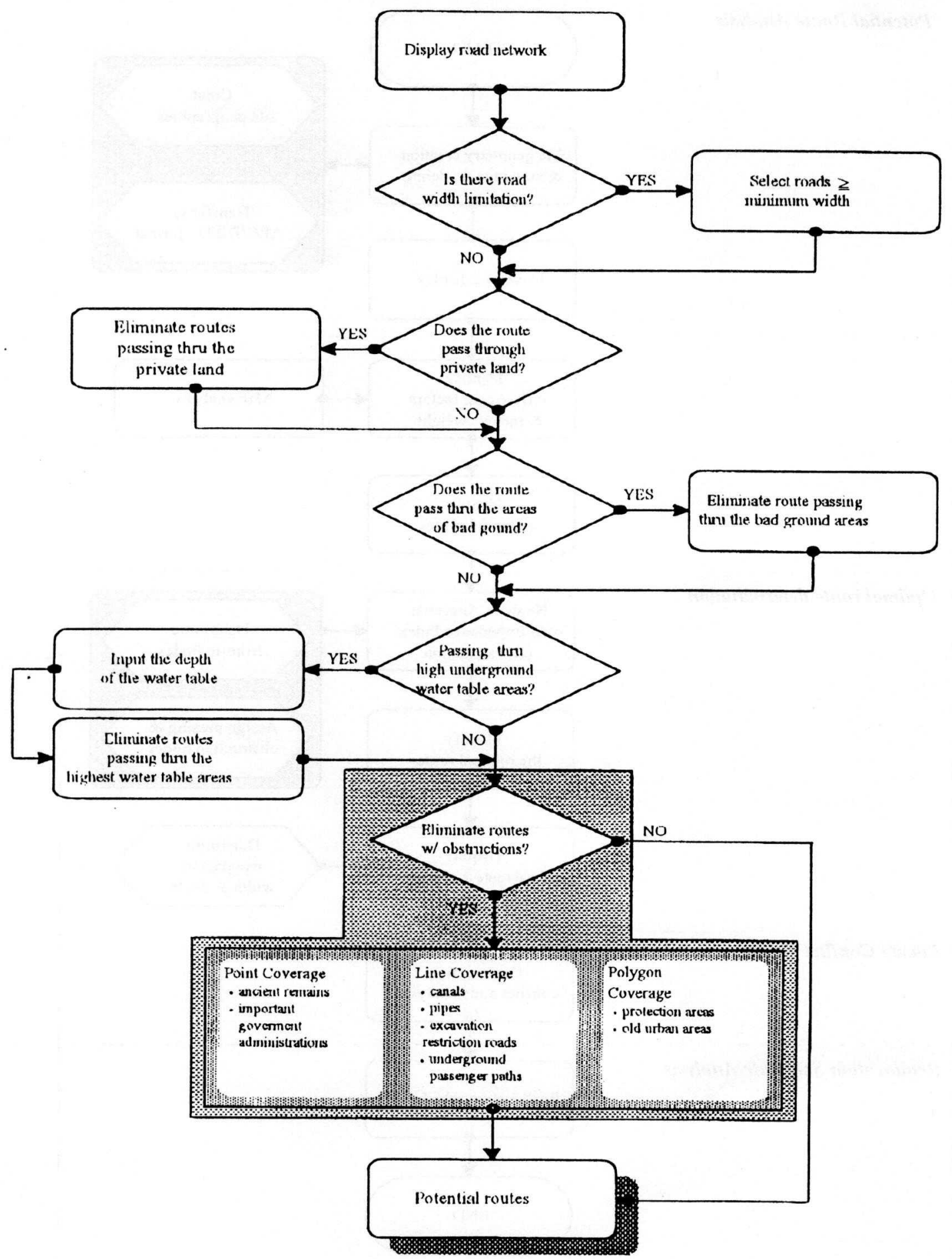

Figure 2 Potential Route Analysis 


\subsection{System Architecture}

The architecture of the system involves identification of the tools used to develop the system functional modules and the means by which each will interface with one another and the user. Figure 3 shows the architecture of the system. The user and program interface for RoutePlan is established at three levels: application user interface, command user interface, and program data interface.

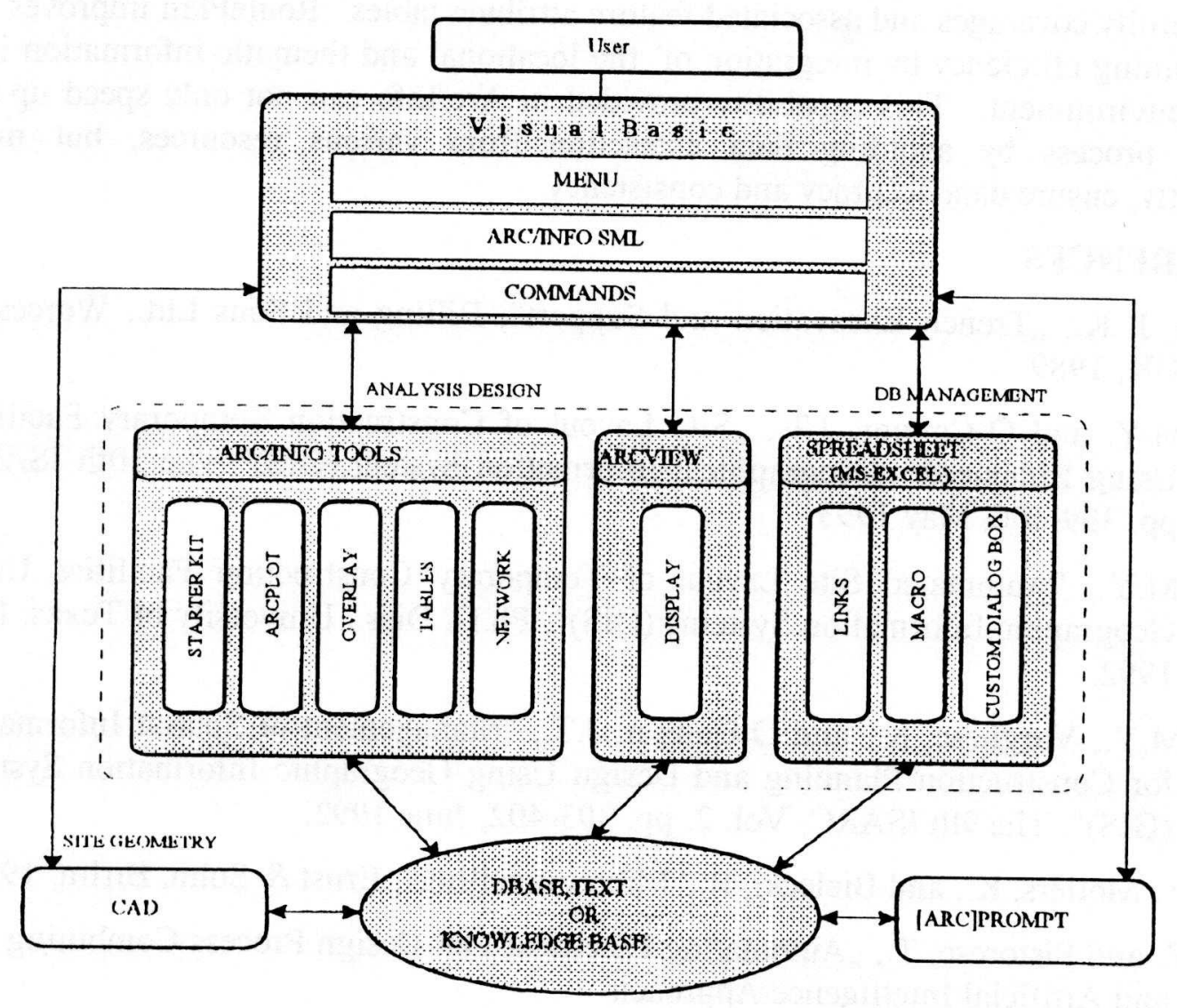

Figure 3 System Architecture

The prime components of the system includes Visual Basic, CAD, Arc/Info, ArcView, Excel spreadsheet, and [ARC] prompt. The user communicates with the components of the system, through a custom interface developed in Visual Basic. The CAD software, AutoCAD is used to create the site geometry. Using the Arc/Info functional modules such as Starter Kit, Arcplot, Overlay, Tables, and Network, the route plan and design is carried out. ArcView is used to perform the graphical query and display the analysis results. Excel spreadsheet is the tool used to add the additional tabular attributes and calculate the impedance index. To enhance the flexibility of the system, the system allows the user to suspend the execution of the SML macros and temporarily exit to the [ARC] prompt to use the Arc/Info functions. 


\section{CONCLUSIONS}

This paper focuses on developing an automated routing system to assist the engineers to select a suitable route to avoid existing obstacles and to minimize the construction cost and schedule. The system, RoutePlan, systematically organize the incomplete and illstructured information regarding the following areas: (1) development of the route selection knowledge base (KB) to represent the expert's knowledge and experience for route planning, (2) application of AHP method to identify the dominant factors for the optimal route selection, and (3) development of the Arc/Info database to contain the existing utility coverages and associated feature attribute tables. RoutePlan improves the route planning efficiency by integration of the locational and thematic information into a single environment. This capability provided in Arc/Info can not only speed up the planning process by avoiding data extraction from various resources, but more importantly, ensure data accuracy and consistency.

\section{REFERENCES}

Budleigh, J. K., „Trench Excavation and Support”, Billing and Sons Ltd., Worcester, UK, 1989

Cheng, M.Y. and O'Connor, J.T., „Site Layout of Construction Temporary Facilities Using Enhanced - Geographical Information System (GIS)" The 10th ISARC, pp. 399-406, May 1993.

Cheng, M.Y., "Automated Site Layout of Temporary Construction Facilities Using Geographic Information Systems (GIS)", Ph.D. Diss., University of Texas, Dec. 1992.

Cheng, M.Y., Varghese, K., and O'Connor, J.T., "Management of Spatial Information for Construction Planning and Design Using Geographic Information Systems (GIS)", The 9th ISARC, Vol. 2, pp. 393-402, June 1992.

Stein, D., Mollers, K., and Bielecki, R.,"Microtunnelling", Ernst \& Sohn, Birlin, 1989.

Sumic, Z. and Pistorese, T., „Automating Electrical Plat Design Process Combining GIS and Artificial Intelligence Approach"

Varghese, K., „Automated Route Planning for Large Vehicles on Industrial Construction Sites", Ph.D. Diss., University of Texas, August 1992. 